\title{
ROSAT HRI catalogue of X-ray sources in the SMC region
}

\author{
M. Sasaki, F. Haberl, and W. Pietsch \\ Max-Planck-Institut für extraterrestrische Physik, Giessenbachstraße, 85748 Garching, Germany
}

Received March 24; accepted August 22, 2000

\begin{abstract}
During the operational phase of the ROSAT satellite between 1990 and 1998 the X-ray telescope pointed 71 times to the Small Magellanic Cloud (SMC) for observations with the High Resolution Imager (HRI), covering a field of $5^{\circ} \times 5^{\circ}$. From these data a catalogue of 121 discrete X-ray sources was derived. By cross-correlating the source catalogue with the SIMBAD data base and the TYCHO catalogue, the systematic positional error of the HRI source positions could be reduced. In total the X-ray position for $99 \mathrm{HRI}$ sources was corrected yielding positional errors between $1^{\prime \prime}$ and $16^{\prime \prime}$. The HRI catalogue was also cross-correlated with the catalogues derived from the ROSAT Position Sensitive Propotional Counter (PSPC) pointings (Kahabka et al. 1999; Haberl et al. 2000). For 75 HRI sources PSPC counterparts were found and thus hardness ratios are given. With the help of the information obtained from the cross-correlations $56 \mathrm{HRI}$ sources were identified with objects of known or proposed nature. Four foreground stars, six supernova remnants, four supersoft sources, 12 $\mathrm{X}$-ray binaries, and one AGN were detected by the HRI. Based on the existence of a likely optical counterpart or properties like hardness ratio and X-ray to optical flux ratio, further 15 HRI sources were classified into different source types.
\end{abstract}

Key words: catalogs — galaxies: magellanic clouds galaxies: stellar content $-\mathrm{X}$-rays: galaxies — X-rays: stars

\section{Introduction}

Since the Magellanic Clouds (MCs) are among the closest galaxies to the Milky Way, we are able to study their overall characteristics such as source distribution as well as properties of single objects within the galaxies.

Send offprint requests to: M. Sasaki;

e-mail: manami@mpe.mpg.de
Complementary to the Large Magellanic Cloud (LMC) source catalogue of the ROSAT High Resolution Imager (HRI) published by Sasaki et al. (hereafter SHP00) HRI data of the Small Magellanic Cloud (SMC) region is analyzed in the work presented here. The SMC with a distance of $\sim 60 \mathrm{kpc}$ has been subject to many studies in different wavelength bands (e.g. Davies et al. 1976; Kennicutt \& Hodge 1986; Staveley-Smith et al. 1997).

X-ray emission from the MCs was first observed in a rocket mission in 1970 (Price et al. 1971). The first discovered single X-ray source in the Small Magellanic Cloud (SMC) was SMC X-1 detected in an Uhuru observation (Leong et al. 1971). The Einstein Observatory performed several X-ray observations of the SMC making a thorough survey (Seward \& Mitchell 1981; Inoue et al. 1983; Bruhweiler et al. 1987; Wang \& Wu 1992). Pointed observations by the X-ray satellite ROSAT (Trümper 1982) covered the MCs in the energy range of $0.1-2.4 \mathrm{keV}$. Kahabka et al. (1999, hereafter KPFH99) presented the first ROSAT PSPC source catalogue of the SMC. After analyzing all the available PSPC pointings Haberl et al. (2000, hereafter HFPK00) published a ROSAT PSPC catalogue of discrete sources in the SMC containing 517 X-ray sources.

\section{ROSAT HRI data}

\subsection{Source detection}

In a $5^{\circ} \times 5^{\circ}$ field around $\mathrm{RA}=01^{\mathrm{h}} 00^{\mathrm{m}} 00^{\mathrm{s}}$, Dec $=$ $-73^{\circ} 00^{\prime} 00^{\prime \prime}$ (J2000.0) including the whole SMC a total of 71 pointings were performed by the ROSAT HRI (David et al. 1996) between 1991 and 1998. The total exposure time is shown in Fig. 1 as contours plotted over a grey scale PSPC image (HFPK00). The number of the analyzed HRI pointings with integration times higher than 100 seconds is demonstrated in Fig. 2.

For data analysis and correction of X-ray positions the same procedures as developed for the LMC (see SHP00) were applied to the SMC data using the EXSAS software package (Zimmermann et al. 1994). For each pointing 


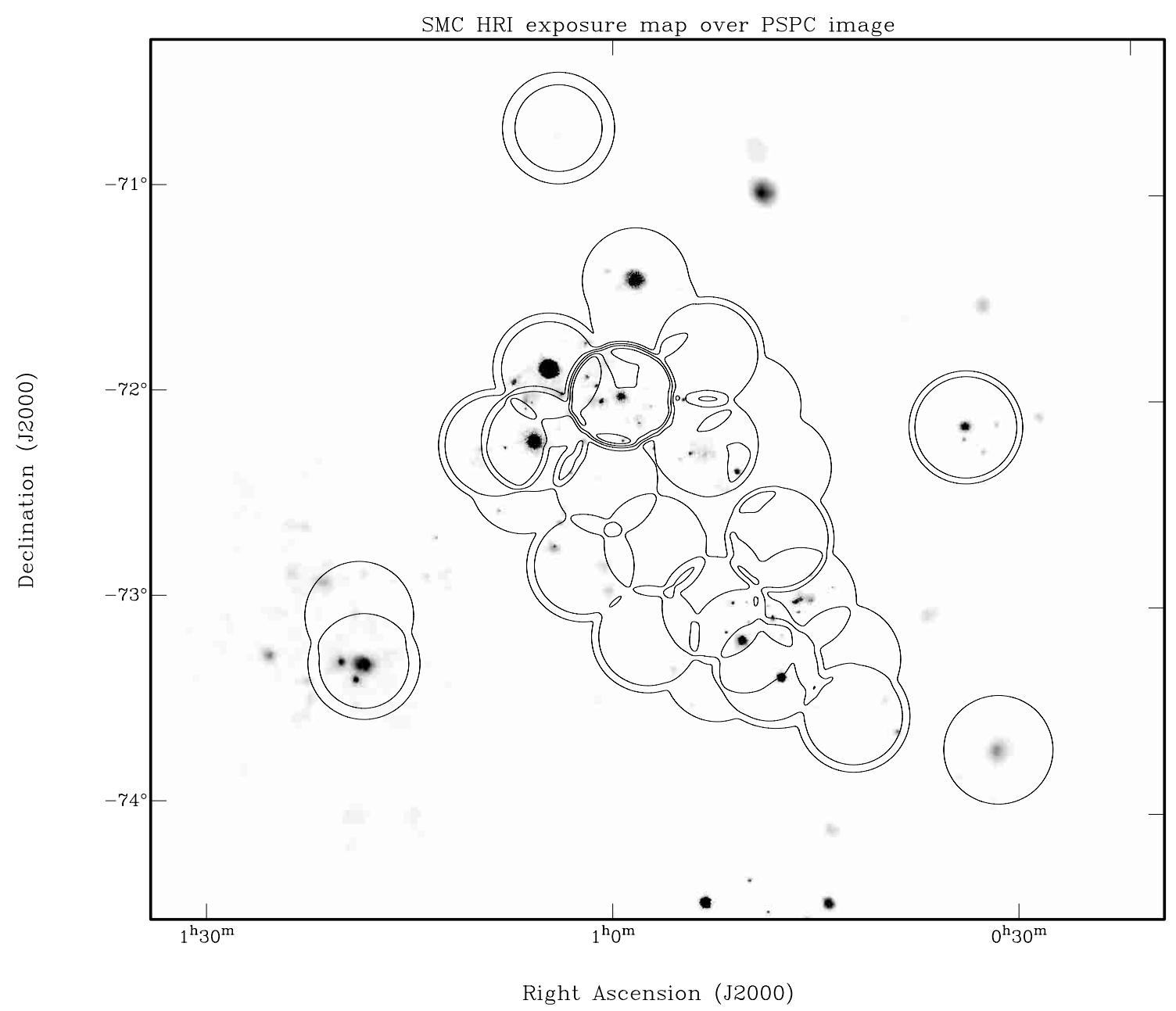

Fig. 1. Contours of total exposure times of the HRI pointings plotted on a grey scale PSPC image $(0.1-2.4 \mathrm{keV})$. The contours signify regions of exposure times with $1,20,40,60,80$, and $100 \mathrm{ksec}$

sources were detected in two sliding window methods using the local background and a spline fitted background map. Both detection lists served as input for the following maximum likelihood method. Detections with existence likelihood higher than 10.0 and telescope off-axis angle smaller than $15^{\prime}$ were accepted as X-ray sources. In addition pointings with directions within a radius of $1^{\prime}$ were merged in order to increase the significance of source signals. For 18 regions in the SMC new deeper data could be created. Further faint sources were found by the source detection procedure in the merged data and augmented the number of detected HRI sources. Finally the source lists from the single and coadded pointings were merged. Multiple detections of one source in different pointings were reduced to one catalogue entry choosing the detection with the highest positional accuracy. In the end we obtained a HRI source catalogue with 121 sources in the SMC region.

This source catalogue was cross-correlated with the PSPC catalogue (HFPK00). We looked for coincidences with objects in the SIMBAD data base operated at the
Centre de Données astronomiques de Strasbourg, the TYCHO catalogue from the ESA Hipparcos space astrometry satellite (Hoeg et al. 1997), and the point source catalogue from the Deep Near Infrared Survey of the Southern Sky (DENIS, Cioni et al. 2000, hereafter CLH00) in order to identify X-ray sources. The sources were also compared to objects known from literature like those published by KPFH99 or Schmidtke et al. (1999). Table 5 lists the HRI sources with corrected coordinates (see Sect. 2.2), positional error, existence likelihood, HRI count rate, extent, extent likelihood, the corresponding PSPC source with count rate and hardness ratios (HFPK00).

\subsection{Source position}

For ROSAT the systematic positional error (boresight error) is about $7^{\prime \prime}$. In cases where an X-ray source in a pointed observation can be identified with an object with accurately known coordinates, the systematic error can be reduced for all detected X-ray sources from the same 


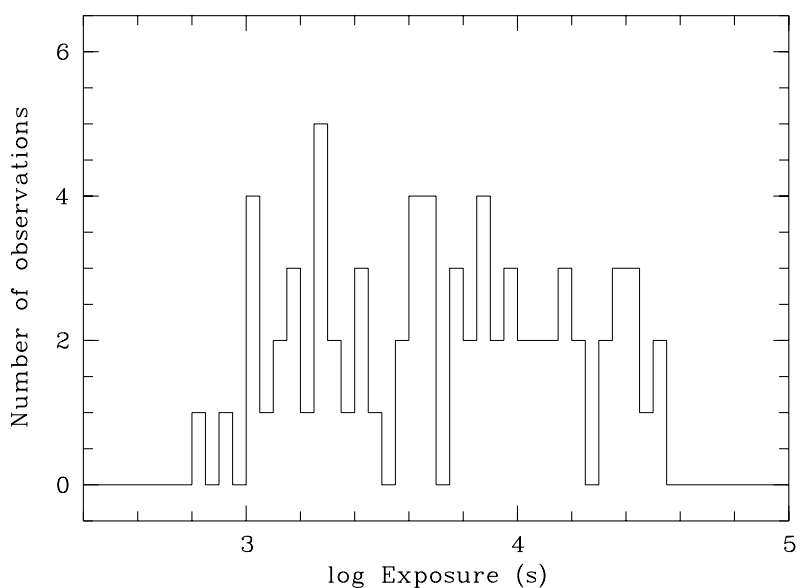

Fig. 2. Histogram for HRI pointing exposure times of SMC observations

observation. The remaining systematic error for all sources in that pointing is

$\sigma_{\mathrm{sys}, \mathrm{p}}=\sqrt{\sigma_{\mathrm{opt}}^{2}+\sigma_{\mathrm{stat}, \mathrm{s}}^{2}}$

$\sigma_{\text {opt }}$ being the error of the optical position and $\sigma_{\text {stat,s }}$ the statistical $90 \%$ confidence error of the identified $\mathrm{X}$-ray source used for correction. A systematic error of $7^{\prime \prime}$ was used for all other sources of which the X-ray position could not be corrected. The total positional error is finally given by:

$\sigma_{\text {tot }, \mathrm{X}}=\sqrt{\sigma_{\text {sys }, \mathrm{p}}^{2}+\sigma_{\text {stat }, \mathrm{X}}^{2}}$.

Therefore the positions of HRI sources identified with TYCHO catalogue sources were corrected to more accurate optical coordinates. For X-ray sources already discussed in the literature and for which the optical counterpart is known, the $\mathrm{X}$-ray positions were also corrected. For each correction the identification of the HRI source with an optical object was confirmed on Digitized Sky Survey (DSS2, red) frames. With the help of the corrected X-ray source positions the coordinates of all the pointings observing these sources were newly computed, and for other sources detected in the corrected pointings again more accurate coordinates were determined. In doing so the X-ray positions of 99 out of 121 sources were improved.

Before the corrections the mean positional error of the 121 HRI catalogue sources was 8"'1. After the boresight correction the mean positional error was reduced to 5.'6, and 4.9 for corrected sources only.

\subsection{Flux variability}

In the maximum likelihood algorithm for source detection, count rates are computed for point sources with intensity distribution peaking in the center. For extended sources the values resulting from the maximum likelihood algorithm are not reliable, because the count rates are

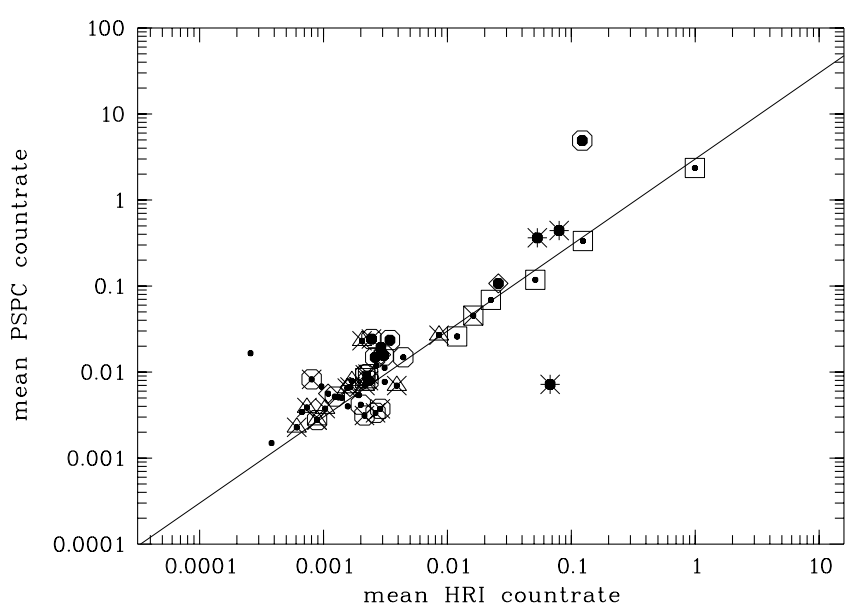

Fig. 3. The mean of observation-averaged count rates from PSPC pointings versus HRI count rates: All sources observed by both detectors are plotted with small dots. In addition SNRs are marked with squares, XBs with circles, SSSs with asterisks, AGN with triangles, and stars with lozenges. Crossed symbols indicate already known candidates and variable sources are additionally marked with larger dots. The line is drawn for a PSPC/HRI conversion factor of 3

determined by fitting a Gaussian to the source intensity profile. Thus for extended sources the total count rate was interactively integrated within a circle around the source, subtracting the background from a ring around the source. For faint sources upper limits were calculated by the maximum likelihood algorithm.

109 HRI sources were detected in more than one ROSAT observation and allowed flux variability studies on time scales of days to years. Lightcurves for these HRI sources were produced in order to test the flux variability. For this purpose not only HRI pointings were used, but also the PSPC pointings of the SMC region. From PSPC count rates and upper limits corresponding HRI values were computed by dividing the PSPC values by 3 which is a typical conversion factor for the ROSAT detectors (see SHP00). Figure 3 shows the correlation between the ROSAT detector count rates for sources detected both by the HRI and the PSPC.

A $\chi^{2}$-test and the ratio between maximum and minimum flux give information on the significance of variability in the lightcurves (HP99a). Variations with reduced $\chi^{2}$ higher than 5.0 were accepted as significant. Finally the list of variable sources contains $16 \mathrm{HRI}$ sources, including three supersoft sources (SSSs) and eight X-ray binaries (XBs) and candidates. Table 1 lists parameters indicating variability and the mean count rates both for $\mathrm{HRI}$ and for PSPC observations for variable HRI sources. 
Table 1. HRI sources with significant flux variability

\begin{tabular}{|c|c|c|c|c|c|c|c|}
\hline 1 & 2 & 3 & 4 & 5 & 6 & 7 & 8 \\
\hline No & $\begin{array}{c}\text { Rate } \\
\text { HRI } \\
{\left[\operatorname{cts~s}{ }^{-1}\right]}\end{array}$ & $\begin{array}{c}\text { Rate } \\
\text { PSPC } \\
{\left[\operatorname{cts~s}^{-1}\right]}\end{array}$ & $\frac{F_{\max }}{F_{\min }}$ & Red. $\chi^{2}$ & DOF & $\begin{array}{c}\text { No } \\
\text { PSPC }\end{array}$ & Remarks \\
\hline 7 & $1.0 \mathrm{e}-01$ & $4.4 \mathrm{e}-01$ & 2.5 & 112.1 & 8 & 176 & SSS 1E0035.4-7230 \\
\hline 10 & $4.4 \mathrm{e}-03$ & $1.6 \mathrm{e}-02$ & 2.0 & 8.1 & 2 & 562 & foreground star HD 3880 \\
\hline 23 & $1.7 \mathrm{e}-02$ & $7.2 \mathrm{e}-03$ & $2.9 \mathrm{e}+01$ & 475.1 & 3 & 512 & SSS RXJ0048.4-7332 \\
\hline 35 & $1.0 \mathrm{e}-02$ & & $3.7 \mathrm{e}+02$ & 14.1 & 3 & & $<$ foreground star $>$ SkKM 62 \\
\hline 44 & $4.9 \mathrm{e}-03$ & $2.4 \mathrm{e}-02$ & $5.5 \mathrm{e}+03$ & 2973.0 & 6 & 453 & HMXB Be/X RXJ0052.1-7319, pulsar [LPM99],[ISC99] \\
\hline 46 & $3.0 \mathrm{e}-03$ & $1.5 \mathrm{e}-02$ & $4.5 \mathrm{e}+02$ & 9.4 & 9 & 94 & HMXB Be/X RXJ0052.9-7158 [CSM97],[SCC99],[HS00] \\
\hline 51 & $2.7 \mathrm{e}-03$ & & $3.1 \mathrm{e}+02$ & 95.0 & 4 & 242 & HMXB? XTEJ0053-724, pulsar [CML98] \\
\hline 65 & $4.3 \mathrm{e}-03$ & $1.9 \mathrm{e}-02$ & 2.4 & 10.4 & 3 & 508 & \\
\hline 74 & $2.2 \mathrm{e}-03$ & $7.9 \mathrm{e}-03$ & $2.5 \mathrm{e}+04$ & 8.7 & 9 & 136 & HMXB? Be/X [HSO0] \\
\hline 79 & $9.4 \mathrm{e}-02$ & $3.6 \mathrm{e}-01$ & 2.6 & 115.5 & 5 & 47 & SSS 1E0056.8-7146 \\
\hline 93 & $2.9 \mathrm{e}-04$ & $<9.1 \mathrm{e}-03$ & $3.5 \mathrm{e}+02$ & 9.9 & 7 & 132 & HMXB Be/X RXJ0101.0-7206 [KP96],[SCB99] \\
\hline 95 & $2.9 \mathrm{e}-03$ & $2.4 \mathrm{e}-02$ & $1.2 \mathrm{e}+01$ & 8.1 & 9 & 159 & HMXB? Be/X [HSO0] \\
\hline 101 & $4.3 \mathrm{e}-03$ & $1.6 \mathrm{e}-02$ & 2.2 & 8.3 & 3 & 143 & HMXB SAXJ0103.2-7209, pulsar [ISC98],[HS94] \\
\hline 114 & $1.6 \mathrm{e}-02$ & & $6.7 \mathrm{e}+01$ & 119.6 & 4 & & \\
\hline 118 & $3.7 \mathrm{e}-01$ & $4.9 \mathrm{e}+00$ & $2.1 \mathrm{e}+02$ & 24762.9 & 8 & 482 & HMXB SMC X-1 \\
\hline 120 & $3.4 \mathrm{e}-02$ & $1.1 \mathrm{e}-01$ & 2.6 & 12.1 & 8 & 478 & foreground star HD 8191 [CSM97] \\
\hline
\end{tabular}

Notes to Cols. Nos. 2 and 3: Count rates are the mean of output values from maximum likelihood algorithm for single pointings. Count rate with $<$ is mean upper limit.

Notes to Col. No. 6: Degrees of freedom.

Notes to Col. No. 7: Source number from HFPK00.

Notes to Col. No. 8: Sources classified in this work are put in $\langle>$. Abbreviations for references in square brackets are given in the literature list.

\section{Source classes}

Various source classes with different origin of the X-ray emission are found in the MC regions: Foreground stars in the Galaxy, supernova remnants (SNRs), SSSs, XBs, and background objects like galaxies and clusters. The X-ray sources detected by the HRI can be assigned to the aforementioned classes if they are identified with known objects from literature (Sect. 3.1) or if their distinguished X-ray properties allow this. Seventy five out of 121 HRI catalogue sources were also detected by the PSPC. We studied the X-ray properties of the identified sources to obtain tools for classifying detections by the HRI (Sect. 3.2).

\subsection{Source identification}

56 HRI sources were identified with known SMC objects, foreground stars, or AGN based on their accurate positions from HRI observations and the X-ray properties like extent or PSPC hardness ratios (HFPK00) as it is shown in Table 3. The accurate HRI positions make it possible to identify likely optical counterparts. For optical sources within the error circle of the X-ray position, the $B$ and $R$ magnitudes were determined from the USNO-A2.0 catalogue produced by the United States Naval Observatory (Monet 1998). The flux ratio was computed from the
$B$ magnitude and the $\mathrm{X}$-ray count rates applying the equation

$$
\begin{aligned}
\log \left(f_{x} / f_{\text {opt }}\right)= & \log \left(3 \cdot \text { HRI counts } / \mathrm{s} \cdot 10^{-11}\right) \\
& +0.4 B+5.37
\end{aligned}
$$

(Maccacaro et al. 1988; HP99b; SHP00). Originally this value was calculated for $V$ magnitude. But since the $V$ magnitude could only be determined for a small sample, we decided to use the $B$ magnitude. It gives a clue for identifying foreground stars, because for stars $\log \left(f_{x} / f_{\text {opt }}\right)$ usually is negative. For very bright stars the $B$ magnitude is not given in the USNO-A2.0 catalogue. In those cases $B$ was set to 11.0 for calculating $\log \left(f_{x} / f_{\text {opt }}\right)$ yielding an upper limit. In Fig. 4 the $\log \left(f_{x} / f_{\text {opt }}\right)$ values are plotted over the PSPC hardness ratio 1 for HRI sources with likely optical counterpart and also detected by the PSPC.

\subsubsection{Foreground stars}

In addition to four foreground stars, two known candidates (sources Nos. 18 and 86) were detected by the HRI. Nos. 18 and 86 were detected by the PSPC and classified as foreground stars by KPFH99 and FHP00. The HRI observations give positions more accurate than the PSPC (total positional error of $5{ }^{\prime \prime} 6$ and $77^{\prime \prime} 2$, respectively). At the HRI positions an optical source was found for each 


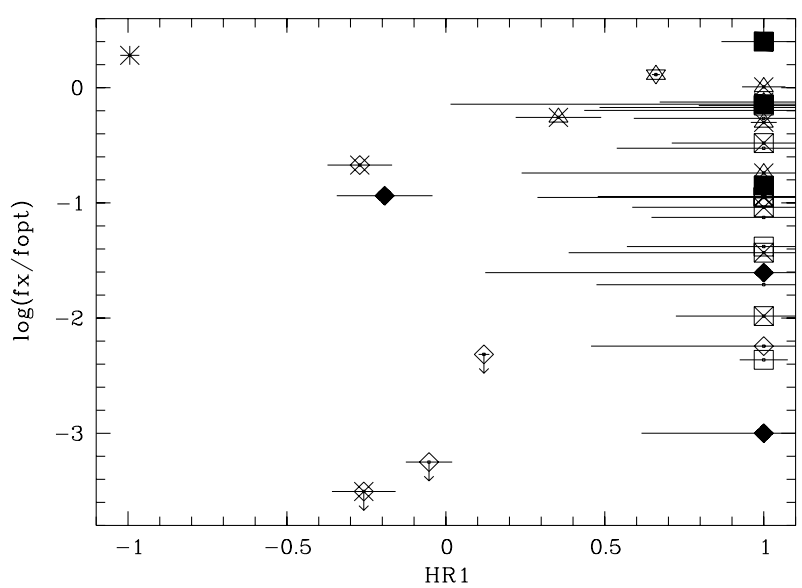

Fig. 4. Flux ratio $\log \left(f_{x} / f_{\text {opt }}\right)$ over hardness ratio 1: Asterisks indicate SSSs, stars SNRs, open squares XBs, open lozenges stars, and open triangles AGN, which are all known objects from literature. Already known candidates are marked with crossed symbols and new classifications with filled symbols

case, with $R=11.3, \log \left(f_{x} / f_{\text {opt }}\right)=-3.51$ for No. 18 and $R=13.1, \log \left(f_{x} / f_{\text {opt }}\right)=-0.67$ for No. 86 confirming the proposed classification.

\subsubsection{Supernova remnants}

Six SNRs were detected by the HRI. For these SNRs extents larger than $5 . \prime 8$ were measured by the HRI with a likelihood higher than 35 (see Fig. 5), except for SNR 0056-72.5 which has no significant extent.

Source No. 22 is identified with a PSPC source which was suggested as SNR candidate by KPFH99. The extent of the HRI source calculated in the maximum likelihood algorithm is only $3{ }^{\prime \prime} 6$. However, the HRI image shows that the source is only the brightest knot in an extended emission, confirming the classification as a SNR candidate.

\subsubsection{Supersoft sources}

Four SSSs were found in the HRI observations which were also detected in PSPC observations. For three of the sources (Nos. 7, 23, and 79) flux variability could be verified by the HRI observations (see Sect. 2.3).

\subsubsection{X-ray binaries}

10 known HMXBs in the SMC were detected both by the PSPC and by the HRI. Two additional HMXBs, AX J0051-722 and SMC X-3, were observed by the HRI only.

Sources Nos. 51 and 73 are HMXB candidates which were observed by other X-ray satellites and are well known from literature. Sources Nos. 39, 81, and 88 were suggested

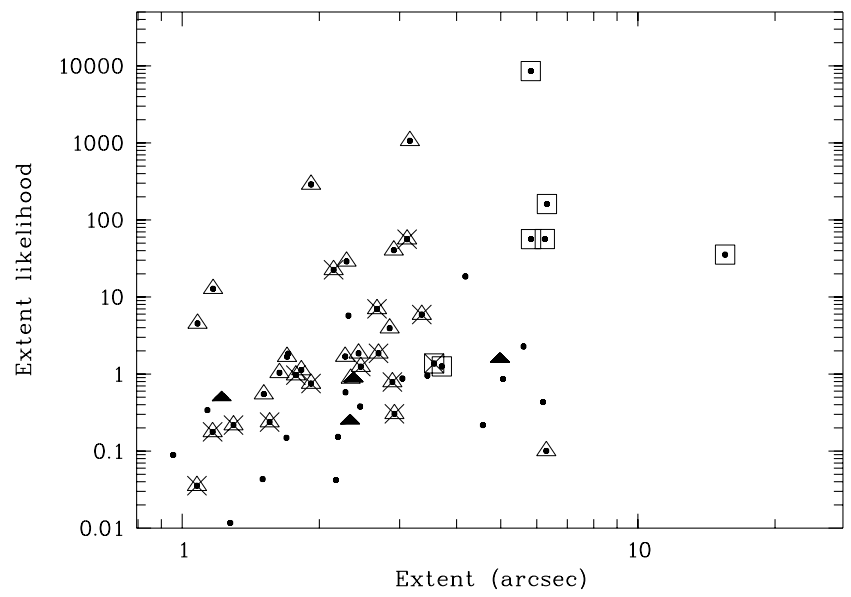

Fig. 5. Source extent is plotted over extent likelihood as small dots for all SMC sources, for which the HRI measured an extent higher than zero. SNRs are additionally marked with squares and known point sources with triangles. Open symbols are used for identified sources, crossed symbols for candidates known from literature, and filled symbols for new classifications

as X-ray binaries by KPFH99. KPFH99 additionally classified the sources Nos. 12 and 83 either as AGN or as $\mathrm{XB}$ candidates. For both there is a likely optical counterpart within the HRI error circle with $B=16.8$ and 17.3, respectively. The hardness ratios are for No. $12 H R 1=$ $1.00 \pm 0.61, H R 2=0.43 \pm 0.16$ and for No. $83 H R 1=$ $1.00 \pm 1.30, H R 2=1.00 \pm 1.93 .10 \mathrm{HRI}$ sources associated with emission line stars (Meyssonnier \& Azzopardi 1993) in the SMC were classified as new HMXB candidates forming Be/X-ray binaries by Haberl \& Sasaki (2000, hereafter HSO0).

\subsubsection{AGN}

Source No. 8 is a known AGN with the redshift $z=0.922$ (Tinney et al. 1997). The hardness ratios are $H R 1=$ $1.00 \pm 1.09, H R 2=-0.06 \pm 0.12$. The optical counterpart seen on DSS2 at the HRI position, which is the same as reported by Tinney et al. (1997), has $B=18.6$ $\left(\log \left(f_{x} / f_{\text {opt }}\right)=-0.49\right)$.

Source No. 59, showing both radio (SMC B0053-7227 (FHW98)) and X-ray emission, was classified as an AGN candidate by KPFH99. A faint optical source $(B=18.6)$ can be found at the HRI position resulting in $\log \left(f_{x} / f_{\text {opt }}\right)$ $=-0.30$. For the source No. 112, also an AGN candidate (FHP00), no optical counterpart was found. The PSPC pointing shows a hard source $(H R 1=1.00 \pm 0.37$, $H R 2=0.46 \pm 0.09)$. Seven HRI sources were classified as AGN candidates by KPFH99. For them likely optical counterparts exist with brightness $17<B<19$. The computed $\log \left(f_{x} / f_{\text {opt }}\right)$ are around -0.50 . 


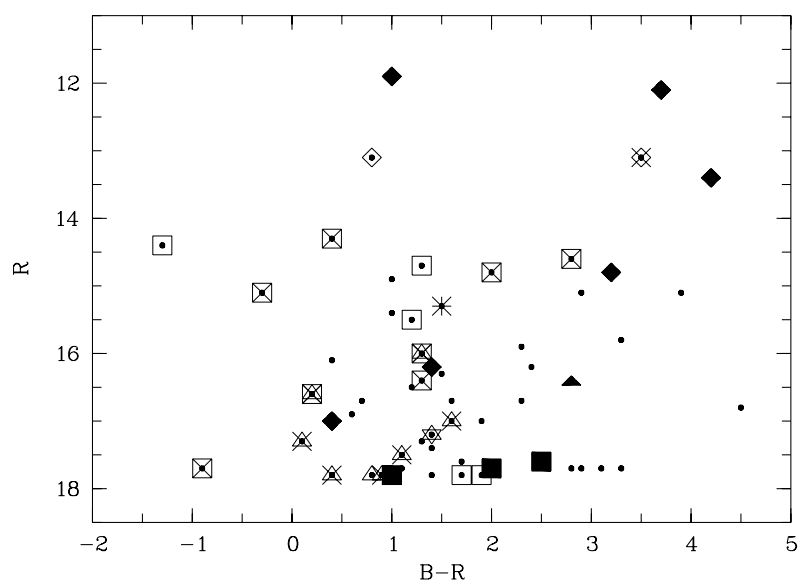

Fig. 6. Color-magnitude diagram for HRI sources correlating with entries in the USNO-A2.0 catalogue. Small dots are used for all sources. Extra asterisks indicate SSSs, stars SNRs, open squares XBs, open lozenges stars, and open triangles AGN. Already known candidates are marked with crossed symbols, so an overlay of open triangle, open square, and cross marks hard sources as candidates either for AGN or XB. Filled symbols are new classifications

\subsection{New classifications}

In addition to the identification of HRI sources with known objects we classified new SMC X-ray sources with the help of their properties obtained from HRI observations. First we looked for extended sources which could suggest new SNR candidates, but without any result. No new source with significant extent was detected.

By comparing positions of X-ray sources with those of stars measured in the optical and near-infrared one can conclude if the X-ray source is in coincidence with a Galactic foreground star. Therefore the HRI source catalogue was cross-correlated not only with the USNO-A2.0 catalogue, but also with the DENIS catalogue, looking for stars within the error circle of the X-ray detection. Hardness ratios determined by PSPC are additional parameters for classifying hard X-ray sources not belonging to the Galaxy as candidates for XBs or AGN.

Table 4 summarizes the new classifications of this work, and in Fig. 8 DSS2 images around these sources are presented with X-ray position and positional error.

\subsubsection{Sources classified as Galactic foreground stars}

In comparison to SMC members or background objects, foreground stars are brighter in $R$ as shown in the colormagnitude diagram (Fig. 6). For the near-infrared CLH00 estimated the number of foreground stars and Magellanic Cloud members in the DENIS catalogue by studying colorcolor and color-magnitude diagrams for the DENIS data. They showed that sources with $J-K_{\mathrm{s}}$ lower than $\sim 1.0$ are most likely foreground stars with increasing probability for
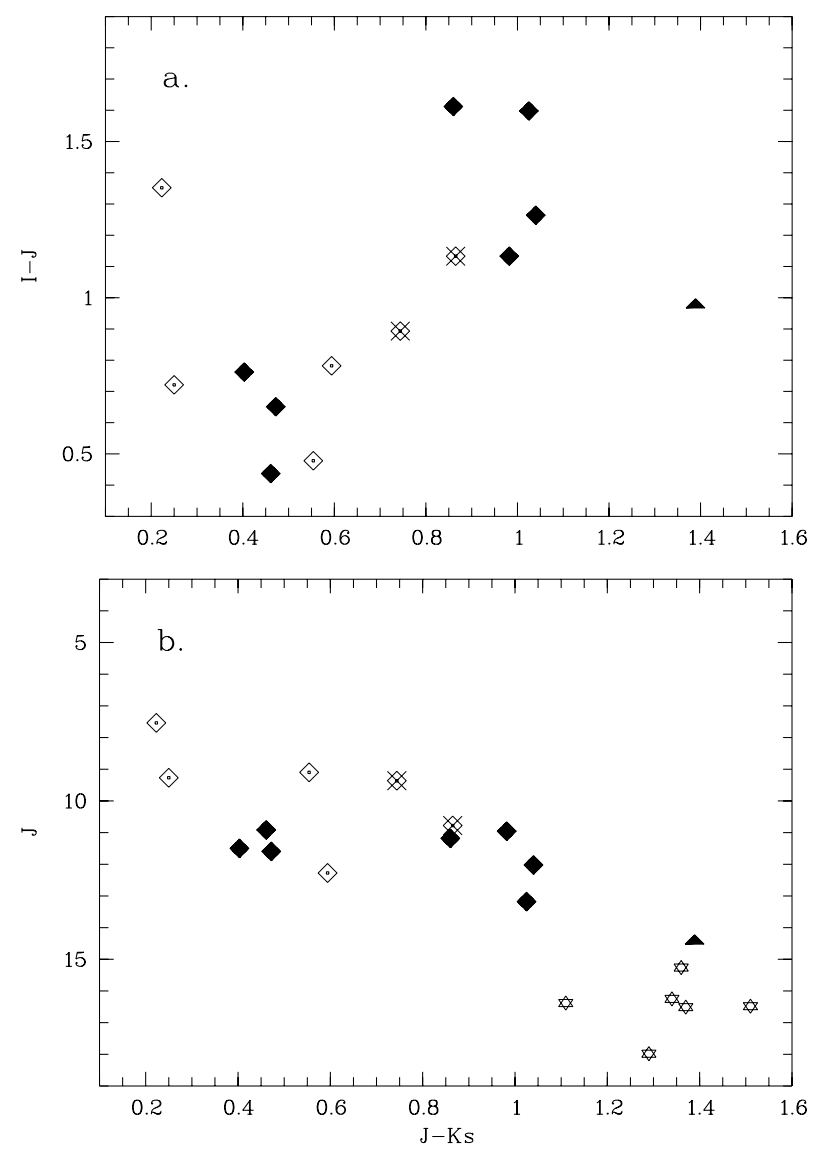

Fig. 7. Color-color and color-magnitude diagram for HRI sources correlating with objects in the DENIS catalogue. Open lozenges for foreground stars, crossed for candidates, and filled for new classifications. The filled triangle mark the source classified as AGN. Stars signify AGN observed by Maiolino et al. (2000)

smaller $J-K_{\mathrm{s}}$ and smaller $I-J$ that such a classification is correct. In our catalogue there are in total 14 sources correlating with entries in the DENIS catalogue. Apart from one, $J-K_{\mathrm{s}}<1.1$ was measured for all of them. Since sources observable both in near-infrared and in $\mathrm{X}$-rays are mainly galactic stars or AGN in the background, seven out of these 13 sources with $J-K_{\mathrm{s}}<1.1$ (Nos. 16, 26, 35, 68, 80, 89, 121) were classified as foreground stars. In Fig. 7a they show a good correlation between $J-K_{\mathrm{s}}$ and $I-J$ which is also shown by CLH00. For most of them we got $R<14.0$ from the USNO-A2.0 catalogue and likely optical counterparts can be seen in the DSS2 images within the error circles (Fig. 8). In addition the source No. 25 was classified as a foreground star since an optical counterpart with $R=10.8$ was found.

\subsubsection{Candidate for AGN}

In the color-color diagram in Fig. 7 there is an outlying source (No. 64). Looking at the color-magnitude diagram 

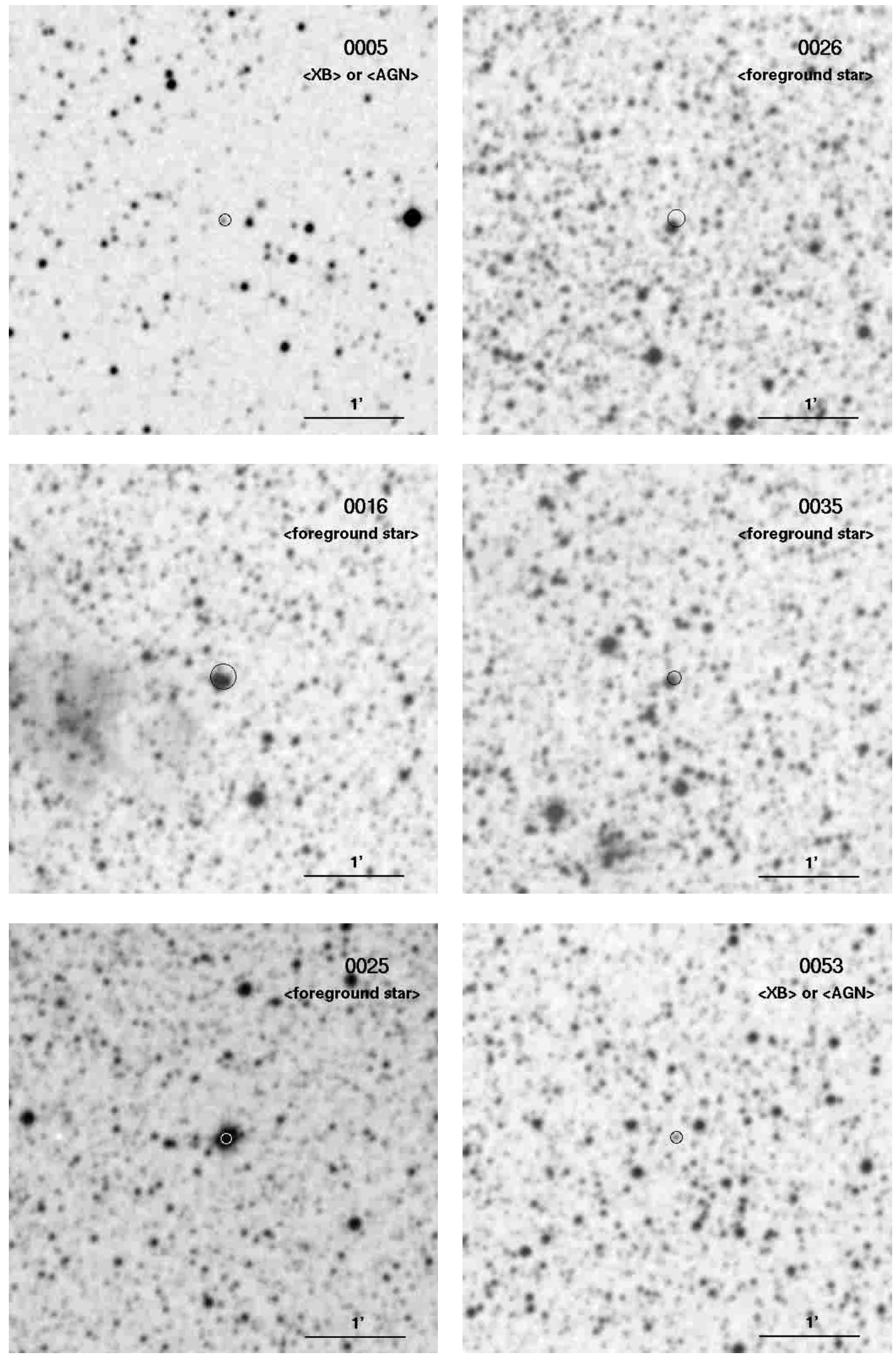

Fig. 8. Positions of newly classified HRI sources on DSS2 images. The comments in brackets $<>$ give the classifications. The $\mathrm{X}$-ray source position is in the center of the image, circles mark the $90 \%$ confidence error 

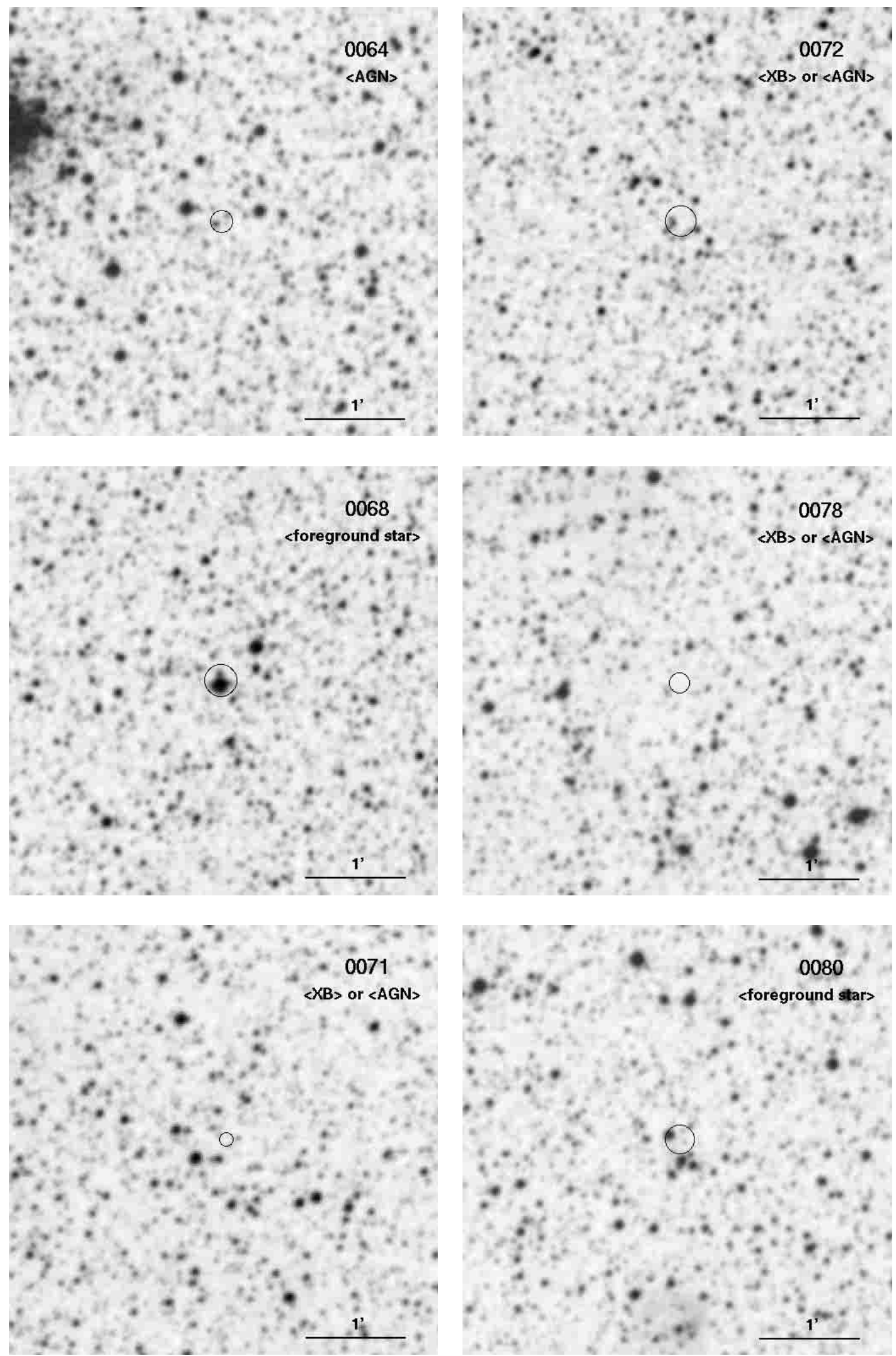

Fig. 8. continued 

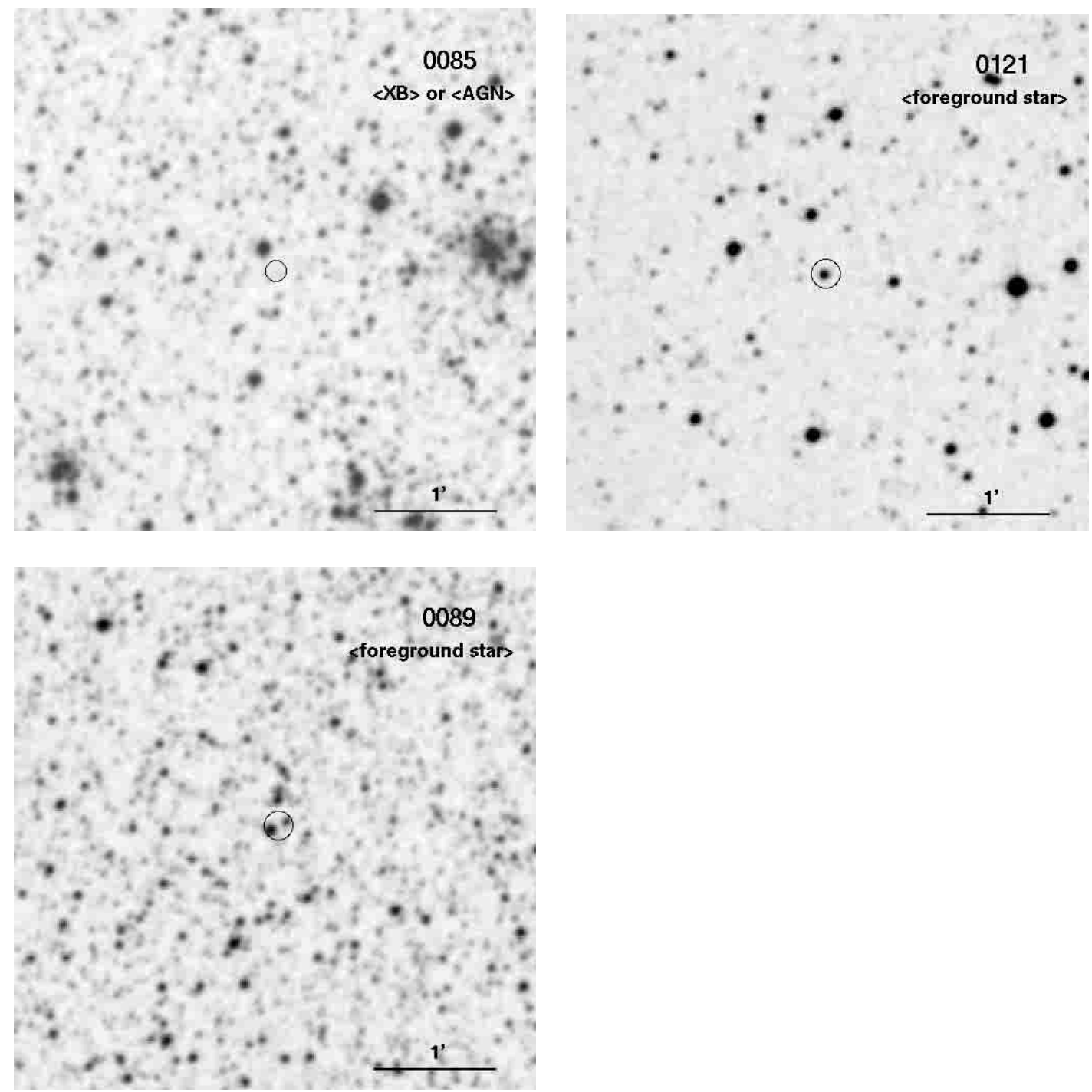

Fig. 8. continued

(Fig. 7b) it is the one with the highest $J-K_{\mathrm{s}}$ and highest $J$. For comparison magnitudes and colors for a sample of AGN determined by Maiolino et al. (2000) are also plotted. The near-infrared and optical brightness of the HRI source No. 68 correspond well with those typical for AGN $(B=19.2, R=16.4)$. So it was classified as an AGN.

\subsubsection{Candidates for AGN or X-ray binaries}

For the source No. 71 no optical counterpart can be found on the DSS2 frame. In the USNO-A2.0 catalogue there is a faint object ( $B=20.1, R=17.6$ ) within the error circle, and with $\log \left(f_{x} / f_{\text {opt }}\right)=0.40$ the HRI source is bright in X-rays. The PSPC spectrum of this source is hard $(H R 1=1.00 \pm 0.13, H R 2=0.38 \pm 0.09)$ favoring its classification as XB or AGN.
Another five HRI sources (Nos. 5, 53, 72, 78, 85) are associated with hard PSPC sources with $H R 1=1.00$ and $H R 2 \geq 0.25$. For all of them large errors were determined for $H R 1$, but not for $H R 2\left(H R 2-\right.$ error $\left._{H R 2}>0.00\right)$, resulting from the fact that the source is so hard that the photon statistics were low in the softer bands (for definition of the hardness ratios see HFPK00). In the USNOA2.0 catalogue only faint or no optical source is found at the HRI position within the error circle. This can be also verified on the DSS2 images (see Fig. 8). Finally these HRI sources were classified as XB or AGN.

\section{Conclusion}

Analyzing the whole set of 71 ROSAT HRI pointings of the SMC region we obtained a catalogue containing 121 discrete X-ray sources. Sources were searched both 


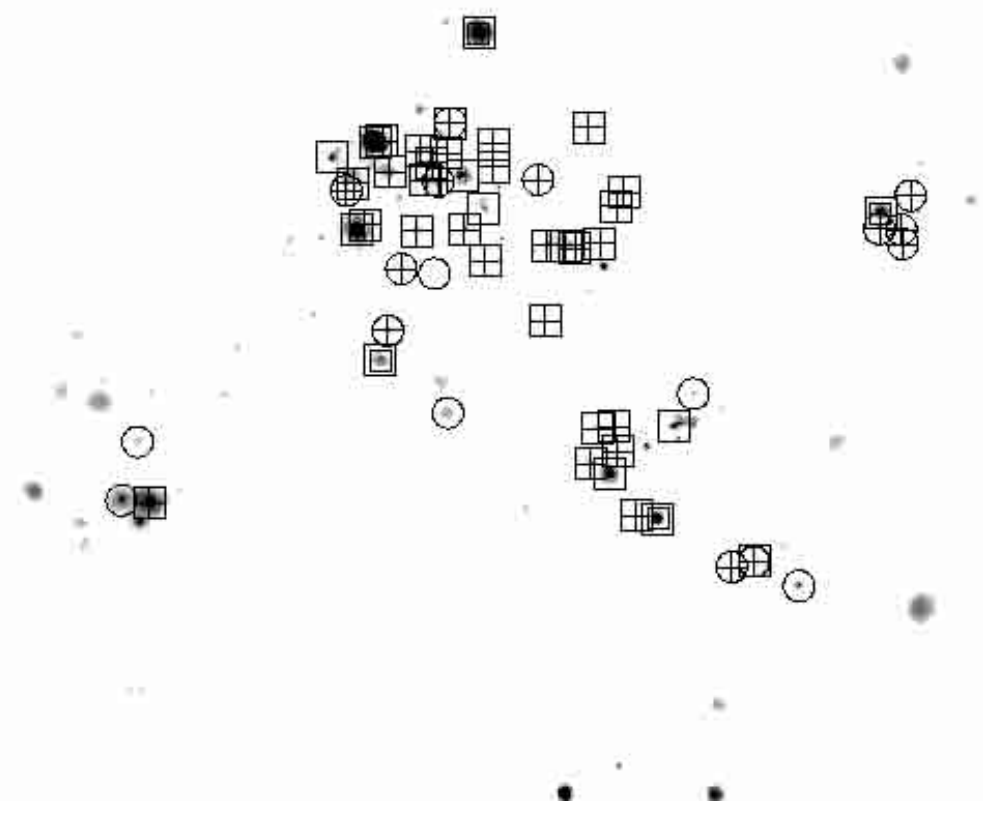

Fig. 9. Distribution of identified HRI sources in the SMC region plotted on a grey scale image $(0.1-2.4 \mathrm{keV})$ obtained from the PSPC data. Squares are used for SNRs, double squares for SSSs, crossed squares for XBs, crossed circles for AGN, circles for foreground stars. Candidates from literature are included for each source class

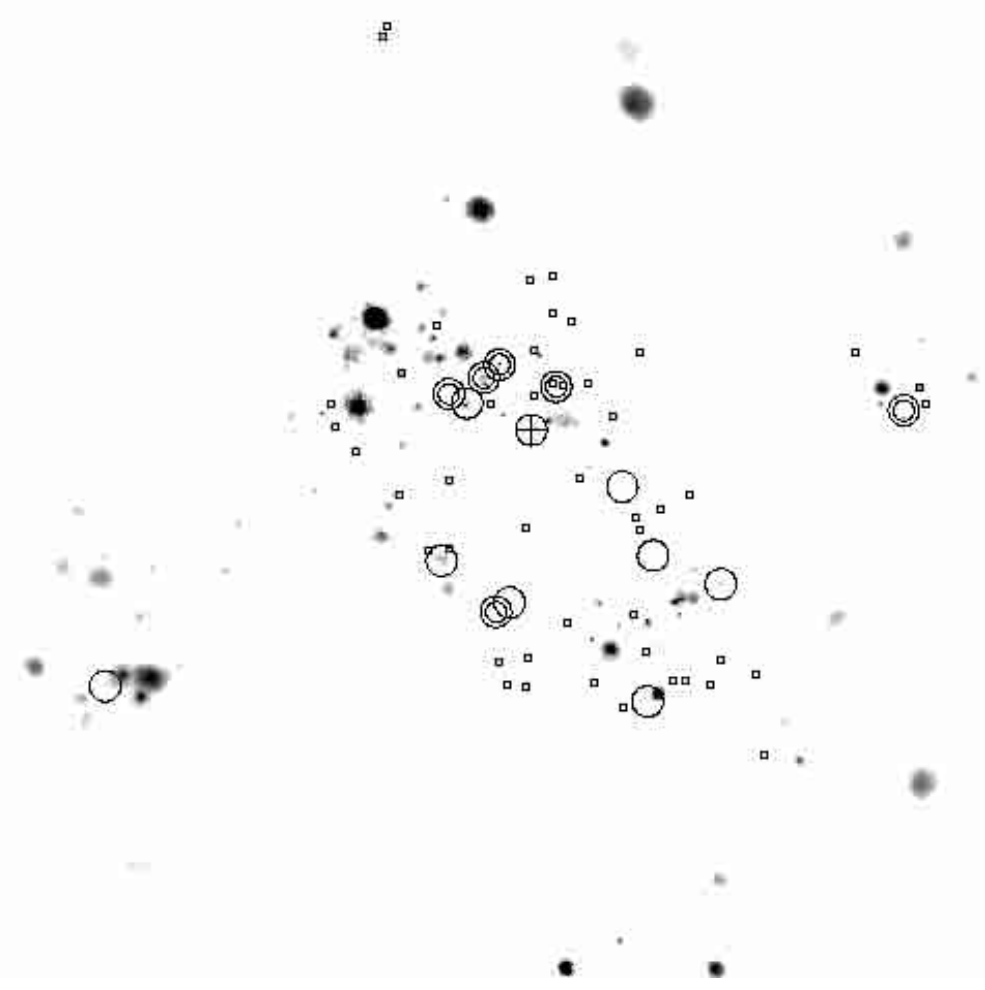

Fig. 10. The distribution of unidentified HRI sources and new source classifications is shown. Unidentified HRI sources are plotted as small squares, circles are classifications as foreground star, crossed circle as AGN, and double circles as AGN or XB candidates 
Table 2. Number of classified X-ray sources in the LMC and SMC observed by the HRI

\begin{tabular}{|l|rr|rr|}
\hline \multicolumn{1}{|c|}{1} & 2 & 3 & 4 & 5 \\
\hline \hline \multicolumn{1}{|c|}{$\begin{array}{c}\text { Source } \\
\text { class }\end{array}$} & LMC & \multicolumn{2}{c|}{ SMC } \\
id.+cand. & new & id.+cand. & new \\
\hline \hline SNR & $24+4$ & 5 & $6+1$ & \\
SSS & 5 & & 4 & \\
XB & $9+1$ & 2 & $12+15$ & \\
AGN & $9+1$ & & $1+9$ & 1 \\
hard (XB or AGN) & & 3 & & 6 \\
foreground star & $39+2$ & & $4+2$ & 8 \\
\hline
\end{tabular}

Notes to Cols. Nos. 2 and 4:

$\mathrm{X}$-ray sources identified with known sources in catalogues and literature + candidates reported in literature.

Notes to Cols. Nos. 3 and 5:

New classifications of SHP00 and this work.

in single pointings and merged data applying a maximum likelihood algorithm. The high spatial resolution of the HRI allowed us to identify X-ray sources with optically observed objects in the TYCHO catalogue or the SIMBAD data base and to reduce the systematic boresight errors. In total coordinates of $99 \mathrm{HRI}$ sources were corrected and the positional errors reduced to 5".6 on average.

By searching through literature 56 HRI sources were identified with already classified objects belonging to SMC SSSs, XBs, SNRs, background AGN, or foreground stars. Figure 9 shows HRI sources which are identified with objects of different source classes known from various catalogues and literature, plotted over an $0.1-2.4 \mathrm{keV}$ PSPC image. The HRI observations mostly covered the main body of the SMC (see Fig. 1), which is the reason that most of the identified and new sources are located in this region (see Fig. 10). In this work 15 sources are newly classified as foreground star, XB, or AGN. There are still 50 unclassified HRI detections well distributed over the field observed by the HRI. The source distribution shows that there is a deficiency of X-ray sources in the central part of the SMC around RA $=00^{\mathrm{h}} 55^{\mathrm{m}}$, Dec $=-72^{\circ} \quad 40^{\prime}$ (J2000.0).

In Table 2 the numbers of detected sources are compared between the LMC and the SMC. In the LMC the number of SNRs is larger than in the SMC by a factor of four roughly in accordance with the ratio of the areas covered by the observations. The number of known SSSs and X-ray binaries detected in ROSAT observations is comparable in the two clouds, most of the XBs are binary systems with Be-star companions. Nevertheless the number of XB candidates in the SMC is relatively higher than in the LMC maybe partly due to the fact that a thorough search for Be/X-ray binary candidates has been done for the SMC by comparing the X-ray catalogues with the catalogues of emission line objects (HS00).

Based on all available ROSAT HRI pointing data complete HRI source catalogues both for the LMC and for the
SMC are now available. They summarize the detections of $\mathrm{X}$-ray sources in the MCs and provide accurate positions with errors smaller than $16^{\prime \prime}$ for all sources, for the majority of sources about $8^{\prime \prime}$. Verification of the candidates for the different source classes by a final optical identification is still needed. Additional observations in the future will achieve more precise characterization of the sources giving a better overall picture of the MCs.

Acknowledgements. The ROSAT project is supported by the German Bundesministerium für Bildung und Forschung (BMBF) and the Max-Planck-Gesellschaft. This research has been carried out by making extensive use of the SIMBAD data base operated at CDS, Strasbourg, France, and data obtained through the High Energy Astrophysics Science Archive Research Center Online Service, provided by the NASA/Goddard Space Flight Center.

\section{References}

Bruhweiler F.C., Klinglesmith D.A., Gull T.R., Sofia S., 1987, ApJ 317, 152

Cioni M.-R., Loup C., Habing H.J., Fouque P., Bertin E., Deul E., Egret D., et al., 2000, A\&AS 144, 235

Corbet R., Marshall F.E., Lochner J.C., Ozaki M., Ueda Y., 1998, IAU Circ. 6803 [CML98]

Cowley A.P., Schmidtke P.C., McGrath T.K., Ponder A.L., Fertig R.M., 1997, PASP 109, 21 [CSM97]

David L.P., Harnden Jr. F.R., Kearns K.E., Zombeck M.V., 1996, The ROSAT High Resolution Imager (HRI), USRSDC/SAO Calibration Report 1996 February, revised Davies R.D., Elliot K.H., Meaburn J., 1976, MmRAS 81, 89

Filipović M.D., Haberl F., Pietsch W., Morgan D., 2000, A\&A 353, 129 [FHP00]

Filipović M.D., Haynes R.F., White G.L., Jones P.A., 1998, A\&AS 130, 421 [FHW98]

Haberl F., Pietsch W., 1999a, A\&A 344, 521 [HP99a]

Haberl F., Pietsch W., 1999b, A\&AS 139, 277 [HP99b]

Haberl F., Filipović M.D., Pietsch W., Kahabka P., 2000, A\&AS 142, 41 [HFPK00]

Haberl F., Sasaki M., 2000, A\&A 359, 573 [HS00]

Hoeg E., Bässgen G., Bastian U., et al., 1997, A\&A 323, L57

Hughes J.P., Smith R.C., 1994, AJ 107, 1363 [HS94]

Israel G.L., Stella L., Campana S., Covino S., Ricci D., Oosterbroek T., 1998, IAU Circ. 6999 [ISC98]

Israel G.L., Stella L., Covino S., Campana S., Mereghetti S., 1999, IAU Circ. 7101 [ISC99]

Inoue H., Koyama K., Tanaka Y., 1983, IAU Symposium 101, Supernova Remnants and Their X-Ray Emission, Danziger J., Gorenstein P. (eds.). Dordrecht: D. Reidel, p. 535

Kahabka P., Pietsch W., 1996, A\&A 312, 919 [KP96]

Kahabka P., Pietsch W., Filipović M.D., Haberl F., 1999, A\&AS 136, 81 [KPFH99]

Kennicutt Jr. R.C., Hodge P.W., 1986, ApJ 306, 130

Lamb R.C., Prince T.A., Macomb D.J., Finger M.H., 1999, IAU Circ. 7081 [LPM99]

Leong C., Kellogg E., Gursky H., Tananbaum H., Giacconi R., 1971, ApJ 170, L67

Maccacaro T., Gioia I.M., Wolter A., Zamorani G., Stocke J.T., 1988, ApJ 326, 680 
Maiolino R., Salvati M., Antonelli L.A., et al., 2000, A\&A 355, L47

Meyssonnier N., Azzopardi M., 1993, A\&AS 102, 451

Monet D.G., 1998, Am. Astron. Soc. Meet., 193, 120.03

Price R.E., Groves D.J., Rodrigues R.M., Seward F.D., Swift C.D., Toor A., 1971, ApJ 168, L7

Sasaki M., Haberl F., Pietsch W., 2000, A\&AS 143, 391 [SHP00]

Schmidtke P.C., Cowley A.P., Crane J.D., Taylor V.A., McGrath T.K., 1999, AJ 117, 927 [SCC99]

Staveley-Smith L., Sault R.J., Hatzidimitriou D., Kesteven M.J., McConnell D., 1997, MNRAS 289, 225
Seward F.D., Mitchell M., 1981, ApJ 243, 736

Stevens J.B., Coe M.J., Buckley D.A.H., 1999, MNRAS 309, 421 [SCB99]

Tinney C.G., Da Costa G.S., Zinnecker H., 1997, MNRAS 285, 111 [TDZ97]

Trümper J., 1982, Adv. Space Res. 2, 241

Wang Q., Wu X., 1992, ApJS 78, 391

Yokogawa J., Koyama K., 1998a, IAU Circ. 6853 [YK98a]

Yokogawa J., Koyama K., 1998b, IAU Circ. 7028 [YK98b]

Zimmermann H.U., Becker W., Belloni T., Döbereiner S., Izzo C., Kahabka P., Schwentker O., 1994, EXSAS User's Guide, MPE report 257 
Table 3. Identified HRI sources in the SMC

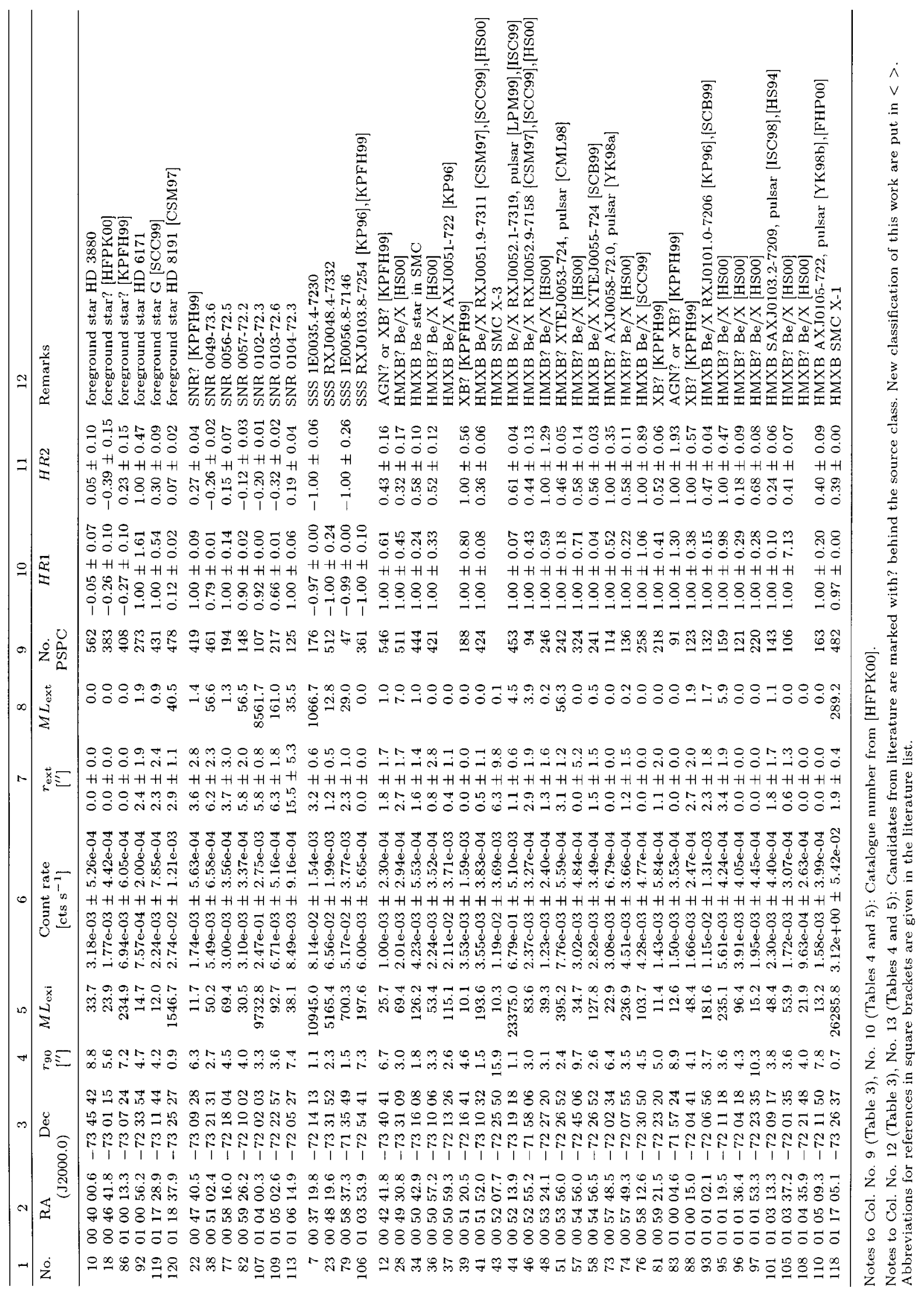


Table 3. continued

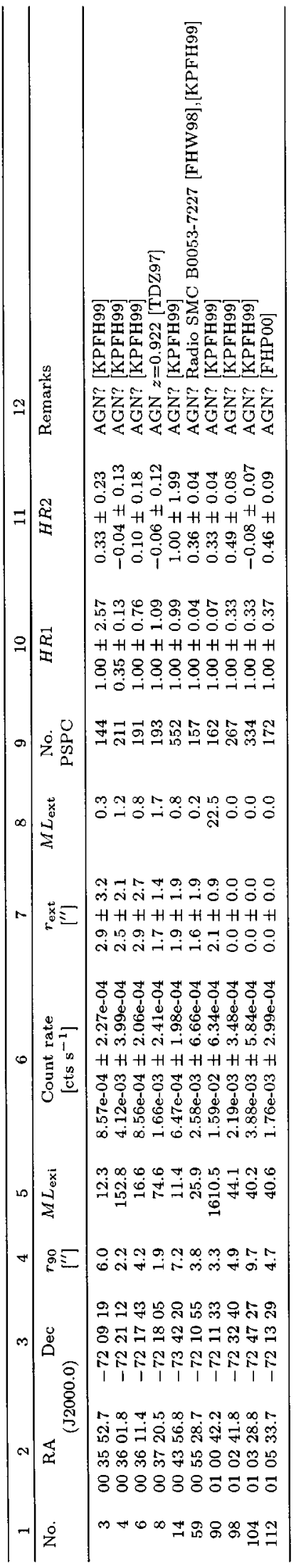

Table 4. Classified HRI sources

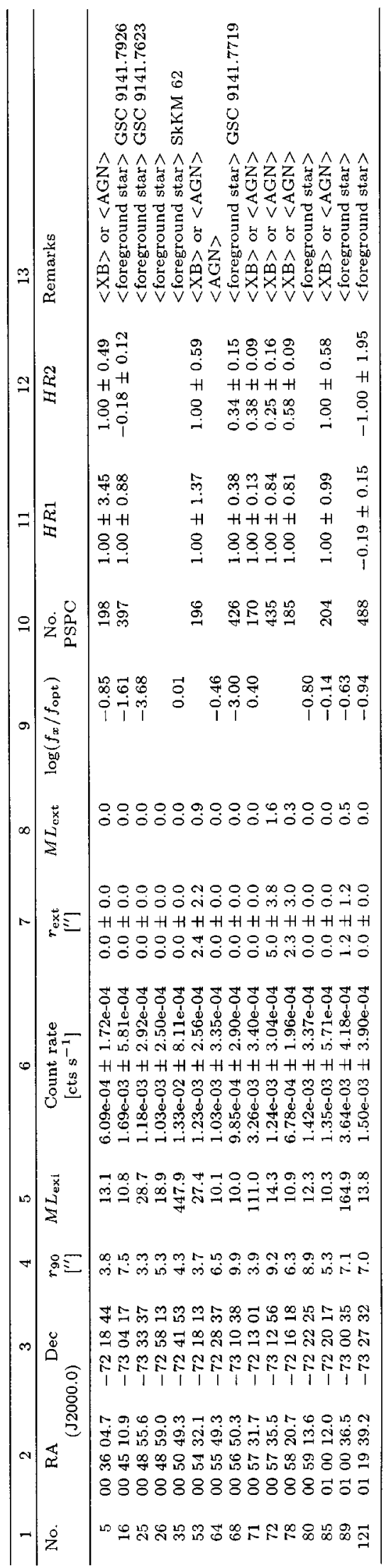


Table 5. HRI sources in the SMC region

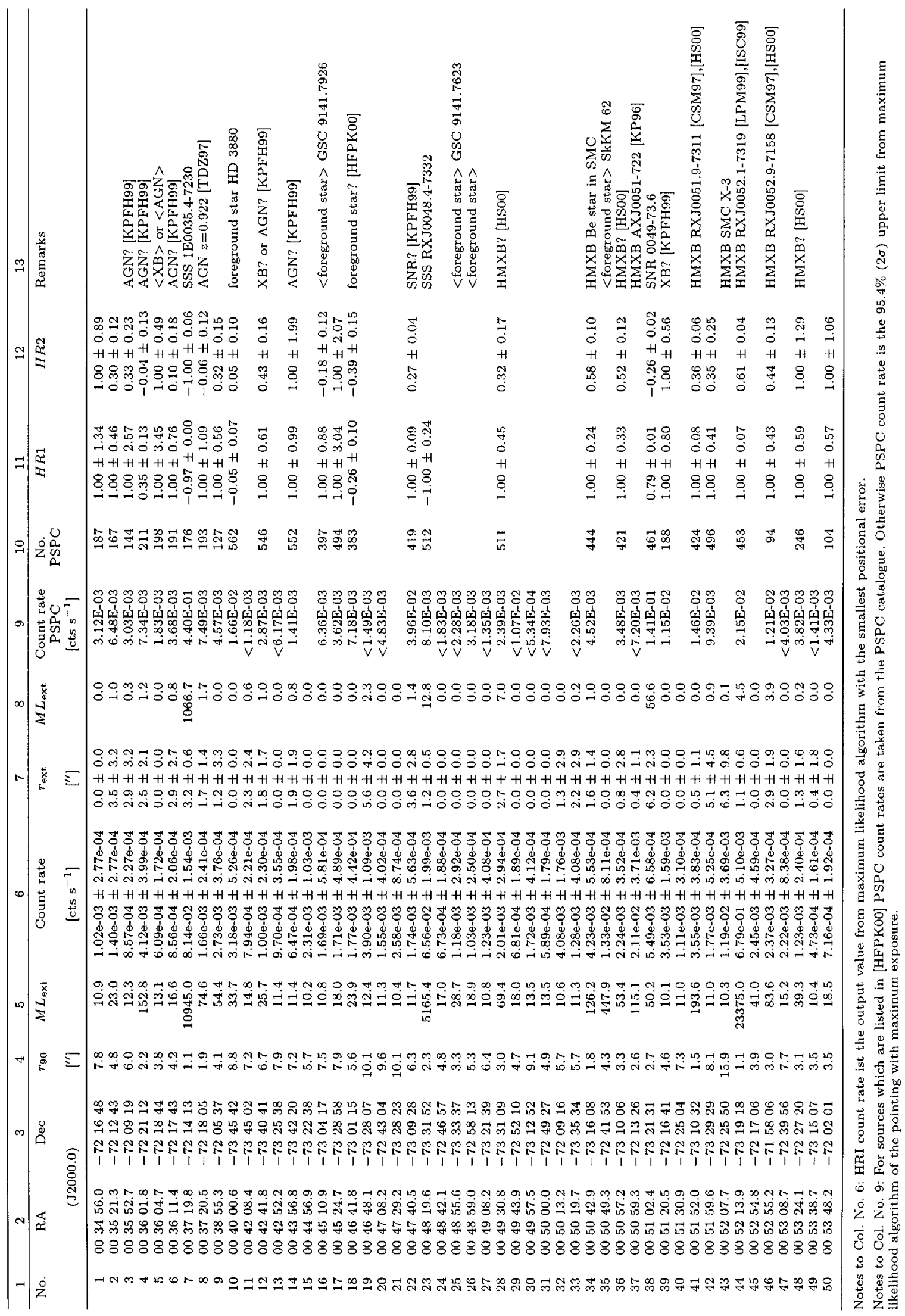


Table 5. continued

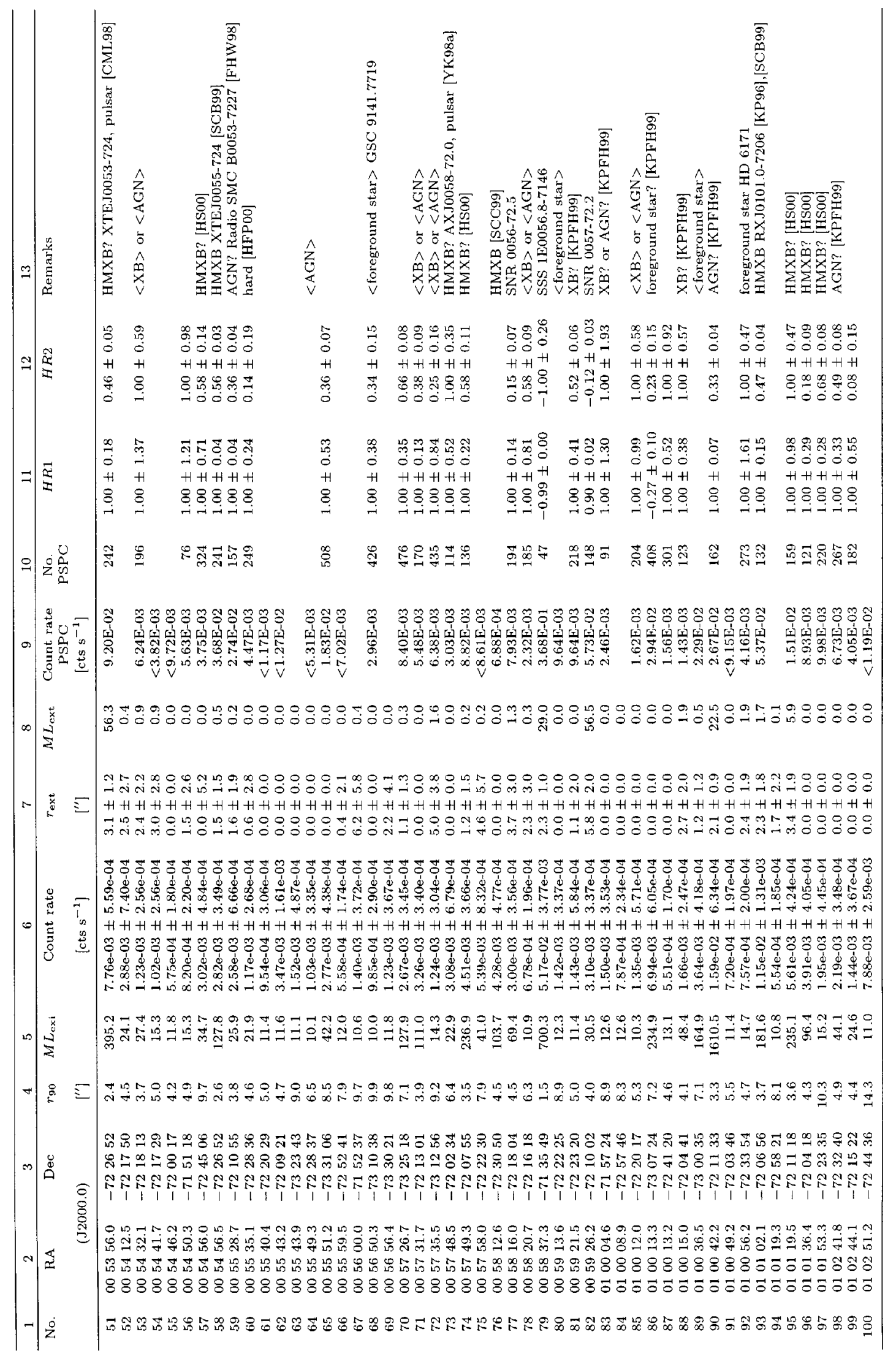


Table 5. continued

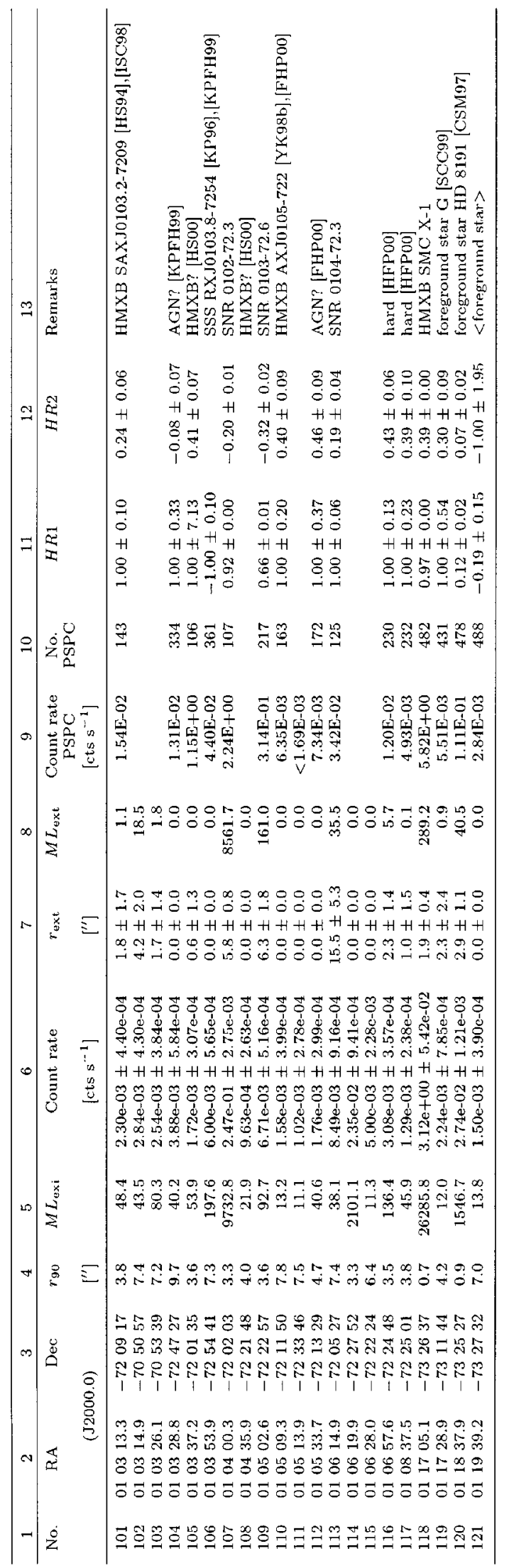

